\title{
Density Functional Theory Investigation on Thiophene Hydrodesulfurization Mechanism Catalyzed by $\mathrm{ReS}_{2}$ (001) Surface
}

Yucheng Huang ${ }^{*}$, Hai Liu, Xi Chen, Danmei Zhou, Chan Wang, Jinyan Du, Tao Zhou, Sufan Wang

Center for Nano Science and Technology, College of Chemistry and Material Science, The Key Laboratory of Functional Molecular Solids, Ministry of Education, Anhui Laboratory of Molecule-Based Materials, Anhui Normal University, Wuhu, 241000, Peoples’ Republic of China

*E-mail: huangyc@mail.ahnu.edu.cn. 
Table S1. Convergence test. Parameters with green background are chosen for the full calculation.

\begin{tabular}{|c|c|c|c|c|c|c|c|c|}
\hline $\begin{array}{l}\text { Cutoff } \\
\text { energy }\end{array}$ & $\begin{array}{c}\text { Energy of } \\
\operatorname{ReS}_{2} \text { bulk } \\
(\mathrm{eV})\end{array}$ & $\begin{array}{l}\text { K-points } \\
\text { for (001) } \\
\text { surface }\end{array}$ & $\begin{array}{c}\text { Energy of } \\
\text { (001) surface } \\
(\mathrm{eV})\end{array}$ & $\begin{array}{l}\text { Cutoff energy } \\
\text { (k-point } 42 \\
1 \text { ) }\end{array}$ & $\begin{array}{c}\text { Energy of (001) } \\
\text { surface }(\mathrm{eV})\end{array}$ & $\begin{array}{c}\text { K-points } \\
(\text { E-cut=400eV) }\end{array}$ & $\begin{array}{l}\text { Thiophene } \\
\text { Adsorption } \\
\text { energy }(\mathrm{eV})\end{array}$ & $\begin{array}{c}\text { Distance of } \\
\text { S-C }{ }_{5} \text { and } \\
\text { S-C }_{2}(\AA)\end{array}$ \\
\hline $\begin{array}{l}200 \\
250\end{array}$ & $\begin{array}{l}-88.628011 \\
-88.941346\end{array}$ & 211 & -357.829452 & 250 & -593.54809821 & 211 & -1.19836125 & 1.742 \\
\hline 300 & -89.127228 & & & 300 & -594.90842964 & & & 1.742 \\
\hline 350 & -89.204378 & 421 & -357.824256 & 350 & -595.48282400 & 421 & -1.255955 & 1.741 \\
\hline $\begin{array}{l}400 \\
450\end{array}$ & $\begin{array}{l}-89.232702 \\
-89.236342\end{array}$ & 631 & -357.824174 & $\begin{array}{l}400 \\
450\end{array}$ & $\begin{array}{l}-595.671431 \\
-595.6726743\end{array}$ & 631 & -1.235269 & $\begin{array}{l}1.740 \\
1.741\end{array}$ \\
\hline 500 & -89.233208 & 841 & -357.824193 & 500 & -595.68992798 & 841 & -1.230468 & $\begin{array}{l}1.737 \\
1.738\end{array}$ \\
\hline
\end{tabular}


Table S2. Imaginary frequencies of each TS (in $\mathrm{cm}^{-1}$ ) along the thiophene HDS pathway catalyzed by the $\operatorname{ReS}_{2}(001)$ surface. The vibrational frequencies are computed with the considering of only thiophenic atoms.

\begin{tabular}{ccccccc}
\hline 1,4-dibutene & $\mathbf{T S}_{1,1}$ & $\mathbf{T S}_{1,2}$ & $\mathbf{T S}_{1,3}$ & $\mathbf{T S}_{1,4}$ & & \\
& 1343 & 1019 & 601 & 453 & & \\
1-butene & $\mathbf{T S}_{234, \mathbf{1}}$ & $\mathbf{T S}_{234,2}$ & $\mathbf{T S}_{234,3}$ & $\mathbf{T S}_{234,4}$ & $\mathbf{T S}_{234,6}$ & $\mathbf{T S}_{2,7}$ \\
& 695 & 1118 & 1150 & 1444 & 742 & 890 \\
2-butene & & & & & & $\mathbf{T S}_{3,7}$ \\
& & & & & & 733 \\
butane & $\mathbf{T S}_{4,7}$ & $\mathbf{T S}_{4,10}$ & $\mathbf{T S}_{4,11}$ & $\mathbf{T S}_{4,12}$ & $\mathbf{T S}_{4,13}$ & \\
& 560 & 846 & 153 & 249 & 781 & \\
\hline
\end{tabular}

